\title{
BRIEF
}

\section{Burnout Among Pharmacy Preceptors in Northern California}

\author{
Laura Baumgartner, PharmD, Lauren Roller, PharmD, Megan LeVay, Jonathan Trinh, PharmD, Amanda \\ Morris, PharmD
}

Touro University California, College of Pharmacy, Vallejo, California

Corresponding Author: Laura Baumgartner, Touro University California, College of Pharmacy, Vallejo, CA 94592. Tel: 608-8526891. Email: Laura.Baumgartner@tu.edu

Submitted June 3, 2021; accepted November 5, 2021; ePublished November 2021

Objective. The objective of this study was to investigate the incidence of burnout syndrome among pharmacy preceptors, and to identify predictors for the development of burnout among this population.

Methods. This cross-sectional survey study examined burnout syndrome among pharmacy preceptors in Northern California. Preceptors were included if they self-identified as a preceptor to Advanced Pharmacy Practice Experience (APPE) students and Pharmacy Graduate Year One (PGY-1) residents. Burnout was assessed using the MBI-HSS, and preceptors were classified as having burnout syndrome if they scored high on emotional exhaustion (EE) plus either high on depersonalization (DP) or low on personal accomplishment (PA). Additionally, demographics, workplace environment, workload, and day-to-day workflow were queried to help determine predictors of burnout syndrome among this population.

Result. One hundred and thirteen pharmacy preceptors were included in the study. Twenty two percent of preceptors reported scores consistent with burnout, with $57 \%$ of preceptors scoring positive for burnout in one of the three burnout criteria. On multivariate regression analysis, two independent risk factors for burnout syndrome were identified; preceptors who precepted a large number of difficult or unmotivated learners per year, and preceptors who did not feel their contributions as preceptors were appreciated by their institution.

Conclusion. The rate of burnout among pharmacy preceptors is high, with preceptors exhibiting high EE and low levels of PA. Precepting a large number of difficult or unmotivated learners, and not feeling contributions as preceptors are appreciated, appear to be predictors of burnout syndrome.

Keywords: burnout, pharmacy, precepting, resident, student

\section{INTRODUCTION}

Burnout among healthcare professionals is an increasing area of concern, with poor well-being and positive burnout being correlated with worse patient safety, including increased medical errors. ${ }^{1}$ In general, prominent characteristics of burnout syndrome include fatigue, poor decision-making abilities, cynical attitude, feelings of inadequacy, and withdrawal from coworkers or patients. ${ }^{1-6}$ The Maslach Burnout Inventory Human Services Survey (MBI-HSS) is the gold standard for assessing burnout among healthcare professionals. This survey characterizes burnout syndrome based on a 3-dimensional-model criterion that includes emotional exhaustion (EE), depersonalization (DP), and personal accomplishment (PA) at work. EE is defined as the stress component of burnout, in which an individual feels depleted of one's emotional and physical resources without any source of replenishment. DP represents an interpersonal dimension of burnout, where an individual displays an indifferent attitude towards their work or their colleagues due to exhaustion. Lastly, PA is measured by the perception of personal accomplishment, which represents the self-evaluation component of burnout and refers to the belief that someone who displays burnout lacks productivity at work. ${ }^{5,7-8}$ Feelings of EE and DP may contribute to burnout, while the perception of PA may serve to offset that.

The incidence and consequences of burnout among physicians and nurses have been well documented in many large trials, while data related to burnout among the pharmacy profession remains limited to select populations. Available literature has described rates of burnout in health-systems pharmacy settings and pharmacy practice faculty, but little is known specifically about pharmacists who serve as experiential preceptors for students and residents. ${ }^{9-13}$ With the expansion in the number of pharmacy schools and residency programs, and the continuing advancement of the pharmacy profession, there has been an increased demand and level of responsibility placed on pharmacist preceptors. The objective 
of this study was to investigate the incidence of burnout among pharmacy student and resident preceptors using the MBIHSS, and to identify predictors for the development of burnout among this population. Determining these key predictors of burnout may help develop strategies to reduce or prevent burnout, and to promote resilience and well-being among pharmacy preceptors.

\section{METHODS}

This was a cross-sectional survey study that was reviewed and approved by the Institutional Review Board (IRB) at Touro University California College of Pharmacy. An invitation to participate in the study was distributed via email to Residency Program Directors (RPDs) at health systems located in Northern California. These health systems were located using a local residency network listerv. RPDs who received the survey invitation were asked to distribute the invitation to participate in an anonymous electronic survey to the pharmacist preceptors at their organization and were also invited to participate in the survey themselves. Selecting the link and choosing to participate served as consent to participate in the study voluntarily. Participants were included in the study if they identified themselves as a preceptor of pharmacy students and pharmacy residents at a Northern California health system. Participants were excluded if they did not complete the entire survey (demographic data and MBI questions) or viewed the link and chose not to participate. The survey data was collected from December 2019 to February 2020. Five \$25 gift cards were offered to be distributed at random as an incentive to promote participation.

Surveys were built using the Qualtrics ${ }^{\mathrm{XM}}$ platform and contained the MBI-HSS embedded at the end of the survey. A Remote Online Survey License for the MBI was purchased prior to enrollment of the study, with a purchase number of two hundred and six copies. The surveys contained 22 statements of job-related feelings from the Maslach Burnout Inventory including nine statements on EE, five statements on feelings of DP, and eight statements on feelings of PA. Participants responded to the job-related feelings statements using a six-point Likert scale to assess how frequently they felt that way. Each Likert scale allowed participants to select a score of zero if they had never experienced that feeling, or a score of six if they felt that way every day. Additionally, demographics, workplace environment, workload (including clinical and precepting responsibilities), and day-to-day workflow were queried.

The primary outcome of the study was to determine the incidence of burnout among pharmacy preceptors. Burnout was defined as a participant having a high EE score plus either high DP or low PA. A participant was considered to have high EE if their sum of EE statements were $>z$, where $z=$ mean $+(S D * 0.5)$ (mean and SD are derived from the EE scores of the entire study population). A participant was considered to have high DP if the sum of their DP statements were $>z$, where $z=$ mean $+\left(S^{*} 1.25\right)$. A participant was considered to have low PA if the sum of their PA statements were $<\mathrm{z}$, where $\mathrm{z}=$ mean $+(\mathrm{SD} * 0.1)$. Additionally, subjects scoring high on EE or DP, or low on PA, were thought to be at an increased risk of burnout syndrome. Although there is no universal definition of burnout using the MBI scoring tool, this study took a more stringent approach by looking at all three aspects, and using the above equation that was adapted by Leiter and Maslach. ${ }^{8}$ The secondary outcome of this study was to determine predictors of burnout among pharmacy preceptors. This included demographic data, preceptor workload, day to day clinical duties, and workplace environment. Statistical analysis:

All data was exported from Qualtrics to Excel v365, and statistical analyses were conducted using STATA for Mac 14.2. As the survey was primarily descriptive in nature, means and standard deviations were reported for continuous data, and frequency and percentages were reported for categorical data. Comparisons were made using a Chi-Square test or Fischer's Exact for categorical data, and Student's t-test for continuous data. Using univariate analysis of burnout, a forward multivariate logistic regression model was created using variables with a p-value $<0.2$. All analyses were conducted with two-sided type I error with alpha 0.05 .

\section{RESULTS}

The survey was distributed to an estimated 300 preceptors located in Northern California, with 113 preceptors completing the survey ( $\sim 37 \%$ response rate). Respondent demographics, and workplace environment, are described in Table 1. The majority of pharmacist preceptors included in this study had been practicing for roughly ten years, were residency trained, and received at least of the Board of Pharmacy Specialty Certifications. In addition, a large percentage of preceptors included in this study practiced at one of the academic health centers in Northern California.

Twenty two percent of pharmacists reported scores consistent with burnout (high EE plus either high DP or low PA), with 5\% of pharmacists scoring positive for all three burnout subscales. Fifty-seven percent of preceptors reported 
scores consistent for burnout in at least one of three subscales. Respondents most frequently scored highly on reduced PA (46\%), followed by feelings of EE (33\%), and DP (12\%) (Table 2).

On univariate analysis, numerous variables were identified as significant predictors of burnout syndrome (Table 1). Forty four percent of preceptors who reported scores consistent with burnout precepted a large percentage of difficult or unmotivated learners per year (26-50\%), versus $16 \%$ of preceptors who did not report scores consistent with burnout ( $\mathrm{p}$ $<0.01)$. In addition, the following variables were associated with less burnout syndrome: working $\leq 40$ hours per week ( $20 \%$ negative burnout vs. $44 \%$ positive burnout, $\mathrm{p}=0.04$ ), working zero weekend shifts per month ( $8 \%$ negative burnout vs. $32 \%$ positive burnout, $\mathrm{p}=0.02)$, feeling as though your contributions as a preceptor were appreciated (44\% negative burnout vs. $78 \%$ positive burnout, $\mathrm{p}<0.01)$, and having incentives at your institution for precepting ( $4 \%$ negative burnout vs. $20 \%$ positive burnout, $\mathrm{p}=0.05$ ). Examples of incentives that preceptors listed were having precepting as part of a career ladder or promotion opportunity, earning precepting hours to take advantage of for bonus or future rotation blocks off, and having educational paid time off.

On multivariate regression analysis, two independent risk factors for burnout syndrome were identified. First, preceptors who precepted a large number of difficult or unmotivated learners per year were significantly more likely to experience burnout syndrome (OR $3.2[1.1-9.6], \mathrm{p}=0.04)$. Secondly, preceptors who felt that their institution appreciated their contributions as preceptors were less likely to experience burnout syndrome (OR 0.32 [0.11 -0.94$], \mathrm{p}=$ $0.03)$.

\section{DISCUSSION}

The current body of literature surrounding burnout in the pharmacy profession is limited, with no studies focusing on burnout among pharmacy preceptors. Burnout among health-system pharmacists and pharmacy practice faculty has been reported to be as high as $61 \%$, which raises the question of burnout among pharmacy preceptors specifically. ${ }^{2,3} \mathrm{With}$ the constant growth of pharmacy schools, and increasing number of residency programs, pharmacists are often tasked with taking on additional precepting roles each year. Preceptors are averaging high numbers of Introductory Pharmacy Practice Experience (IPPE) students, Advanced Pharmacy Practice Experience (APPE) students, and resident learners per year, often without time off from precepting. The increasing number of learners assigned to preceptors, and expanding demands of clinical pharmacy roles, begs the need for literature examining burnout among this specific subset of pharmacists.

The incidence of burnout among pharmacy preceptors in our study is similar to rates of burnout seen in previously published literature examining health-system pharmacists and pharmacy practice faculty. ${ }^{2,3,6,13}$ Durham and colleagues examined burnout among health-system pharmacists, and similarly found that $53.2 \%$ of their participants reported a high degree of burnout on at least one subscale of the MBI-HSS, with $8.5 \%$ of participants indicating burnout across all three subscales. Similar to our findings, Durham and colleagues found that pharmacists tend to show high levels of EE and low levels of PA (average scores were $22.9 \%, 6.2 \%$, and $36.3 \%$ for feelings of EE, DP, and low PA, respectively). ${ }^{3}$ In addition, a study examining the incidence of burnout among pharmacy practice faculty found a similar high degree of burnout. Using a burnout definition of high EE, El-Ibiary and colleagues found that $41.3 \%$ of faculty exhibited high EE, similar to $33 \%$ of preceptors in our study showing signs of EE. ${ }^{13}$

We also aimed to identify potential predictors of burnout among pharmacy preceptors to help institutions develop strategies that may help reduce or prevent burnout and promote well-being among preceptors. While controlling for other factors, two predictors for burnout were identified. These included preceptors who dealt with many unmotivated or difficult learners and preceptors who did not feel as though their institution appreciated their contributions. Although not significant on multivariate regression analysis, having precepting incentives in place for preceptors appeared to be protective of burnout. These findings suggest that creating a workplace environment where preceptors feel valued and appreciated may help lead to less burnout.

The predictors of burnout among pharmacy preceptors identified in this study adds to the current body of literature that examines burnout among health-system pharmacists and faculty members. Jones and colleagues conducted a study looking solely at burnout among inpatient hospital pharmacists, and identified the following factors to be contributory towards burnout: inadequate administrative and teaching time, uncertainty of health care reform, too many nonclinical duties, difficult pharmacist colleagues, and feeling that contributions are underappreciated. ${ }^{2}$ Similar to our findings, both subsets of pharmacists experienced less burnout if they felt their contributions were appreciated by their institution. In addition, when taken together with our results, time spent teaching learners outside of clinical duties, particularly those learners who are difficult or unmotivated, appears to exacerbate burnout. And lastly, when looking at 
predictors of burnout among pharmacy practice faculty, high EE was associated with being female, of the assistant professor rank, and those without a hobby. ${ }^{13}$ Although not statistically significant, our study also found that a higher percentage of the pharmacy preceptors who reported scores consistent with burnout were full time faculty at the assistant professor rank ( $40 \mathrm{vs} 22 \%, \mathrm{p}=0.07)$. Identifying these potential predictors of burnout may help institutions mitigate burnout moving forward, in addition to providing resources for preceptors on how to manage burnout.

This study is not without limitations, however. Although surveys were distributed to roughly 300 pharmacy preceptors in Northern California, there was a $37 \%$ response rate, and we relied on the participants to self-identify as preceptors. It is also worth noting that the results of this study are only representative of pharmacy preceptors in one region of the United States, and in addition the majority of preceptors included in the study were employed by a single academic health center, which may have different precepting demands than other institutions. Lastly, our results may have a component of selection bias. Distribution of the survey to preceptors at the various health systems may not have been unified. In addition, preceptors who were experiencing burnout when the survey was released may have refrained from voluntarily taking the time needed to complete the survey, which could underestimate the incidence of burnout in this study.

\section{CONCLUSION}

The rate of burnout among pharmacy preceptors was high, with preceptors exhibiting high EE and low levels of PA. Significant predictors of burnout among preceptors included precepting a large number of difficult or unmotivated learners per year, and not feeling as though their institution appreciated their contributions as preceptors. Future studies should aim to survey preceptors across all states, in addition to examining the effect various interventions have on reduction of burnout.

\section{REFERENCES}

1. Hall LH, Johnson J, Watt I, et al. Healthcare staff wellbeing, burnout, and patient safety: a systematic review. PloS One. 2016; 11:e0159015

2. Jones GM, Roe NA, Louden L, et al. Factors associated with burnout among us hospital clinical pharmacy practitioners: results of a nationwide pilot survey. Hosp. Pharm. 2017; 52: 742-751.

3. Durham ME, Bush PW, Ball AM. Evidence of burnout in health-system pharmacists. AJHP 2018; 75: S93 S100.

4. Maslach C, Jackson SE, Leiter MP, et al. MBI Manual. $3^{\text {rd }}$ ed. Palo Alto, CA: Consulting Psychologists Press; 1996: 191-218.

5. Bridgeman PJ, Bridgeman MB, Barone J. Burnout syndrome among healthcare professionals. AJHP 2018; 75 : 147-152.

6. Barnett CW, Hopkins WA, Jackson RA. Burnout experienced by recent pharmacy graduates of Mercer University. AJHP 1986; 43: 2780-2784.

7. Maslach C, Schaufeli WB, Leiter MP. Job burnout. Ann Rev Psychol. 2001; 52:397-422

8. Leiter MP, Maslach C. Latent burnout profiles: A new approach to understanding burnout experience. Burn. Res 2016; 3: 89-100.

9. Welp A, Meier LL, Manser T. Emotional exhaustion and workload predict clinician-rated and objective patient safety. Front Psychol. 2015; 5:1573.

10. Shanafelt TD, Boone S, Tan L et al. Burnout and satisfaction with work-life balance among US physicians relative to the general US population. Arch Intern Med. 2012; 172:1377-1385.

11. Canadas-De la Fuente G, Vargas C, San Luis C et al. Risk factors and prevalence of burnout syndrome in the nursing profession. Int J Nurs Stud 2015; 52: 240-249

12. Thomas NK. Resident Burnout. JAMA 2004; 292: 2880 - 2889.

13. El-Ibiary SY, Yam L, Lee KC. Assessment of Burnout and Associated Risk Factors Among Pharmacy Practice Faculty in the United States. AJPE 2017; 81: 1-9.

Table 1. Characteristics of Survey Population

Positive Burnout ${ }^{+}$

$(\mathbf{n}=25)$

\section{Negative Burnout ${ }^{+}$ \\ $(\mathrm{n}=\mathbf{8 8})$}

p- value* 
Age (years)

Gender (male), n (\%)

Relationship Status, n (\%)

Single

Stable Partner

Married

Children/Dependents, n (\%)

Post-Graduate Training, n (\%)

PGY1

PGY2

Board of Pharmacy Specialty Certification, $n(\%)$

Practice Setting, n (\%)

Academic Health Center

Non-University Affiliated Teaching Hospital

Non-Teaching Community Hospital

Veterans Affairs (VA)

Faculty Status, n (\%)

None

Adjunct

Assistant

Associate

Professor

Number of Years as Practicing Pharmacist

Number of Years as a Preceptor

Payment Structure, n (\%)

Hourly (earns overtime)

Salary

Average Hours Worked Per Week

$$
\begin{aligned}
& \leq 40 \\
& 41-50 \\
& 51-60 \\
& >60
\end{aligned}
$$

Average Weekends Worked per Month, n (\%)

$$
0
$$

1

$\geq 2$

Time spent per day rounding/patient visits (hours)

Time spent per day discussing patient care with learners (hours)

Time spent per day on teaching activities (hours)

Number of IPPE^ ${ }^{\wedge}$ Students Precepted per Year

Number of APPE\& Students Precepted per Year

Number of PGY1/PGY2 Residents Precepted per Year

Percentage of learners who are difficult or unmotivated and required additional coaching, $\mathrm{n}(\%)$

$<10 \%$

$10-25 \%$

$26-50 \%$

$>50 \%$

Do you feel your contributions as a preceptor are appreciated by your institution? (yes, $\mathrm{n}(\%)$ )

Does your institution offer incentives for precepting learners? (yes, n (\%))
$35.7 \pm 7.5$

$10(40)$

7 (28)

3 (12)

15 (60)

7 (28)

23 (92)

13 (52)

23 (92)

$20(80)$

3 (12)

2 (8)

0

9 (36)

4 (16)

10 (40)

0

2 (8)

$9.1 \pm 6.8$

$7.7 \pm 5.6$

17 (68)

8 (32)

5 (20)

14 (56)

5 (20)

1 (4)

$2(8)$

12 (48)

11 (44)

$3.4 \pm 2.1$

$2 \pm 1.5$

$1.3 \pm 0.7$

$3.9 \pm 9$

$5.4 \pm 4.8$

$3 \pm 3.2$

2 (8)

12 (48)

11 (44)

0 (0)

11 (44)

1 (4)
$36.1 \pm 7$

28 (32)

0.84

0.48

33 (38)

5 (6)

0.77

50 (57)

37 (42)

0.28

0.78

0.2

82 (93)

42 (48)

1

71 (81)

0.82

0.24

60 (68)

15 (17)

12 (13)

1 (1)

46 (52)

8 (9)

19 (22)

11 (13)

4 (5)

$9.6 \pm 6.5$

$8 \pm 4.8$

0.25

0.76

0.45

1

0.18

0.46

0.07

0.12

0.61

0.71

0.79

60 (68)

28 (32)

$$
1
$$

39 (44)

37 (42)

12 (14)

0

0.04

0.26

0.54

0.33

28 (32)

0.02

28 (32)

0.14

32 (36)

0.49

$3.6 \pm 3$

0.8

$1.7 \pm 1.3$

0.36

$1.5 \pm 1.3$

0.56

$2.8 \pm 5.3$

0.46

$5.1 \pm 4.4$

0.79

$2.9 \pm 3.3$

0.91

20 (23)

0.15

46 (52)

0.82

14 (16)

$<0.01$

8 (9)

0.2

69 (78)

$<0.01$

18 (20)

0.05 
Does your institution offer preceptor development? (yes, $\mathrm{n}(\%))$

Does your institution provide any type of tool or program

${ }^{+}$Positive burnout was defined as a participant having a high EE score plus either high DP or low PA

* Chi-Square test or Fischer's Exact tests were used for categorical data, and Student's t-test for continuous data to determine significance, defined as $\mathrm{p}<0.05$

${ }^{\wedge} \mathrm{IPPE}=$ Introductory Pharmacy Practice Experience

${ }^{\&} \mathrm{APPE}=$ Advanced Pharmacy Practice Experience

Table 2. Logistic Regression Model for Predictors of Burnout

(a)

Variable

OR $[95 \% \mathrm{CI}]$

p-value

Assistant Professor Appointment

$1.52[0.51-4.57] \quad 0.45$

$\leq 40$ Hours Worked per Week

$0.4[0.12-1.39] \quad 0.15$

Zero Weekends Worked

$0.29[0.06-1.52]$

0.14

26-50\% Difficult or Unmotivated Students Who

$3.2[1.1-9.6]$

0.04

Required Additional Coaching

Preceptors felt their contributions were

$0.32[0.11-0.94]$

0.03

appreciated by their institution

Incentives Offered for Precepting

$0.32[0.04-2.78]$

0.3

Table 3. Burnout Categorized by Emotional Exhaustion, Depersonalization, and Personal Accomplishment

\section{Burnout Indices}

Emotional Exhaustion

- Low score $(<=18)$

- Moderate Score

- $\quad$ High Score $(>=31)$

Depersonalization

- $\quad$ Low score $(<=6)$

- Moderate Score

\section{Number (\%)}

$33(29)$

$43(38)$

37 (33)

$61(54)$

38 (34) 
- High Score (>=12)

$14(12)$

Personal accomplishment

- Low score $(<=37)$

- Moderate score

$46(41)$

- High score $(>=44)$ 